\title{
Exactly solvable quantum spin ladders associated with the orthogonal and symplectic Lie algebras
}

\author{
M.T. Batchelor ${ }^{a}$, J. de Gier ${ }^{a}$, J. Links ${ }^{b}$ and M. Maslen ${ }^{a}$ \\ ${ }^{a}$ Department of Mathematics, School of Mathematical Sciences, \\ The Australian National University, Canberra ACT 0200, Australia \\ ${ }^{b}$ Centre for Mathematical Physics, Department of Mathematics, \\ The University of Queensland, QLD 4072, Australia
}

\begin{abstract}
We extend the results of spin ladder models associated with the Lie algebras $s u\left(2^{n}\right)$ to the case of the orthogonal and symplectic algebras $o\left(2^{n}\right), s p\left(2^{n}\right)$ where $n$ is the number of legs for the system. Two classes of models are found whose symmetry, either orthogonal or symplectic, has an explicit $n$ dependence. Integrability of these models is shown for an arbitrary coupling of $X X$ type rung interactions and applied magnetic field term.
\end{abstract}


The study of quantum spin ladders has recently been the focus of both theoretical and experimental investigations [四]. From the theoretical point of view, many researchers are now applying the procedures of the Quantum Inverse Scattering Method (QISM) and associated Bethe Ansatz techniques in order to obtain non-perturbative results for models of spin ladder systems. Originally this was undertaken in [2, 3] by using the co-algebra structure of the Yangian algebra (in fact a Hopf algebra) which underlies the applicability of the QISM. In essence the co-algebra mapping allows the one-dimensional model to be mapped to multiple copies by way of a homomorphism. As such, the algebraic properties are preserved and consequently integrability is maintained, allowing for analyses of these models by standard methods.

Subsequently, an alternative view point has been adopted in which the extension from the one-dimensional system to the ladder model is accomodated by an extension of the symmetry of the system. By such an approach, the ladder is considered as a quasi onedimensional system whereby the rung interactions of the ladder are represented as local symmetry operators. In this manner, the rung interactions may be coupled to the bulk ladder model without violating integrability. This in turn again facilitates the application of many known techniques that have been developed in the rich field of integrable models in order to determine ground state properties, elementary excitation spectra and phase diagrams.

One of the first works in this latter direction is that of Wang [4] in which an integrable formulation of a two-leg spin 1/2 model was given in terms of the maximal local (i.e. rung space) symmetry algebra of $s u(4)$. In this model rung interactions are coupled with arbitrary strength and a phase diagram was obtained in terms of the coupling parameter. In the same paper another model based on $s u(3 \mid 1)$ supersymmetry was also presented and it became apparent that such an approach could be extended to incorporate other forms of symmetry. In [5] it was demonstrated that the natural extension to $n$-leg models gave rise to $s u\left(2^{n}\right)$ symmetry. Other models have also been considered using a "non-standard" solution of the Yang-Baxter relation [6, 7] and others based on su(4) and su(2|2) symmetry [8] in the context of fermion ladder systems. Extension of this approach to a $t-J$ ladder model which is also of significant physical interest can be found in [9].

In the spirit of [5] our goal here is to identify $n$-leg ladder models arising through the orthogonal and symplectic Lie algebras. We find two distinct classes which are integrable for arbitrary coupling of $X X$ rung interactions and magnetic field terms. A curious feature of the models we obtain is that the symmetry algebra has an explicit dependence on the number of legs of the system.

Throughout, we will express all operators in terms of the Pauli matrices

$$
\sigma^{x}=\left(\begin{array}{ll}
0 & 1 \\
1 & 0
\end{array}\right), \quad \sigma^{y}=\left(\begin{array}{cc}
0 & -i \\
i & 0
\end{array}\right), \quad \sigma^{z}=\left(\begin{array}{ll}
1 & 0 \\
0 & 1
\end{array}\right)
$$

which act on a two-dimensional space that we denote $V$. Furthermore, we use a superscript $(l)$ to denote an operator acting on the leg labelled by $l$ and use subscripts to label the rungs.

In order to construct a model on an $n$-leg ladder we begin by introducing a metric for 
the $2^{n}$-dimensional space $W=V^{\otimes n}$ by

$$
\begin{aligned}
\alpha & =\sigma^{x} \otimes \sigma^{y} \otimes \sigma^{x} \otimes \sigma^{y} \otimes \ldots \otimes X \\
& =\prod_{l \text { odd }}\left(\sigma^{x}\right)^{(l)} \prod_{l^{\prime} \text { even }}\left(\sigma^{y}\right)^{\left(l^{\prime}\right)}
\end{aligned}
$$

where $X=\sigma^{x}$ for an odd number of legs and $\sigma^{y}$ for the even case. The metric has the useful properties,

$$
\alpha=\alpha^{-1}=\alpha^{\dagger}=(-1)^{[n / 2]} \alpha^{t},
$$

with $[n / 2]$ being the integer part of $n / 2$. For any basis $\left\{v_{i}\right\}_{i=1}^{2^{n}}$ of $W$, the set of matrices

$$
A_{j}^{i}=e_{j}^{i}-\alpha e_{i}^{j} \alpha,
$$

with the elementary matrices $e_{j}^{i}$ satisfying $e_{j}^{i} v^{k}=\delta_{j}^{k} v^{i}$, in fact close to form a Lie algebra. The commutation relations amongst the generators read

$$
\left[A_{j}^{i}, A_{l}^{k}\right]=\delta_{j}^{k} A_{l}^{i}-\delta_{l}^{i} A_{j}^{k}+\alpha_{k}^{i} \alpha_{m}^{l} A_{j}^{m}-\alpha_{j}^{l} \alpha_{k}^{m} A_{m}^{i}
$$

with implied summation over the repeated index $m$. For the instance where $[n / 2]$ is even these matrices realize the Lie algebra $o\left(2^{n}\right)$ while in the odd case it is $\operatorname{sp}\left(2^{n}\right)$.

As in the $g l\left(2^{n}\right)$ invariant models we begin with the observation that the permutation operator on $W_{i} \otimes W_{j}$ is expressible in terms of the Pauli matrices by

$$
P_{i j}=\frac{1}{2^{n}} \prod_{l=1}^{n}\left(I+\sigma_{i}^{(l)} \cdot \sigma_{j}^{(l)}\right) .
$$

We now introduce a Temperley-Lieb operator [10],

$$
Q_{i j}=(I \otimes \alpha) P^{t_{j}}\left(I \otimes \alpha^{t}\right),
$$

with the properties,

$$
\begin{aligned}
Q^{2} & =(-1)^{[n / 2]} 2^{n} Q \\
Q_{i(i+1)} & =Q_{i(i+1)} Q_{(i+1)(i+2)} Q_{i(i+1)} \\
Q_{i(i+1)} & =Q_{i(i+1)} Q_{(i-1) i} Q_{i(i+1)}
\end{aligned}
$$

This operator on $W_{i} \otimes W_{j}$ reads, when expressed in Pauli matrices,

$$
Q_{i j}=\frac{(-1)^{[n / 2]}}{2^{n}} \prod_{l=1}^{n}\left(I-\sigma_{i}^{(l)} \cdot M^{(l)} \sigma_{j}^{(l)}\right),
$$

where the matrix $M^{(l)}$ is diagonal with entries

$$
M^{(l)}=\operatorname{diag}\left((-1)^{l},(-1)^{l}, 1\right) .
$$

It is well known that given any Temperley-Lieb operator there is an associated integrable model. The operator defined by

$$
\check{R}(u)=I+\frac{(-1)^{[n / 2]} \sinh u}{\sinh (\gamma-u)} Q,
$$


gives a solution of the Yang-Baxter equation,

$$
\check{R}_{12}(u) \check{R}_{23}(u+v) \check{R}_{12}(v)=\check{R}_{23}(v) \check{R}_{12}(u+v) \check{R}_{23}(u),
$$

where the parameter $\gamma$ is determined by

$$
\cosh \gamma=2^{(n-1)}
$$

Furthermore, the set of operators $\left\{G_{i}=(-1)^{[n / 2]} P_{i(i+1)}, E_{i}=Q_{i(i+1)}\right\}_{i=1}^{L-1}$ together give a representation of the Birman-Wenzl-Murakami algebra [11]. Using the results of [12], we can now obtain the operator

$$
\check{R}(u)=I+u P-\frac{u}{u+2^{(n-1)}-(-1)^{[n / 2]}} Q,
$$

which also gives a solution of the Yang-Baxter equation (12).

By the standard approach, integrable models with periodic boundary conditions of the form

$$
H=\sum_{i=1}^{L-1} H_{i(i+1)}+H_{L 1},
$$

can be obtained from the above solutions of the Yang-Baxter equation, where the local Hamiltonians $H_{i j}$ read

$$
H_{i j}=\left.\frac{d}{d u} \check{R}_{i j}(u)\right|_{u=0} .
$$

For the Temperley-Lieb models we obtain

$$
\begin{aligned}
H_{i j} & =\frac{(-1)^{[n / 2]}}{\sinh \gamma} Q_{i j} \\
& =\frac{1}{2^{n} \sinh \gamma} \prod_{l=1}^{n}\left(I-\sigma_{i}^{(l)} \cdot M^{(l)} \sigma_{j}^{(l)}\right),
\end{aligned}
$$

while in the Birman-Wenzl-Murakami case we have

$$
\begin{aligned}
H_{i j} & =P_{i j}-\frac{1}{2^{(n-1)}-(-1)^{[n / 2]}} Q_{i j} \\
& =\frac{1}{2^{n}} \prod_{l=1}^{n}\left(I+\sigma_{i}^{(l)} \cdot \sigma_{j}^{(l)}\right)-\frac{(-1)^{[n / 2]}}{2^{n}\left(2^{(n-1)}-(-1)^{[n / 2]}\right)} \prod_{l=1}^{n}\left(I-\sigma_{i}^{(l)} \cdot M^{(l)} \sigma_{j}^{(l)}\right) .
\end{aligned}
$$

By our construction, the above Hamiltonians are invariant with respect to the algebra elements defined by (4). Additionally, we can couple to these models $X X$ rung interactions and applied magnetic field terms

$$
\frac{1}{2} J \sum_{i=1}^{L} \sum_{l=1}^{n-1} h_{i}^{(l)}+\frac{1}{2} B \sum_{i=1}^{L} \sum_{l=1}^{n} g_{i}^{(l)}
$$


where

$$
\begin{aligned}
h_{i}^{(l)} & =\left(\sigma^{x}\right)_{i}^{(l)}\left(\sigma^{x}\right)_{i}^{(l+1)}+\left(\sigma^{y}\right)_{i}^{(l)}\left(\sigma^{y}\right)_{i}^{(l+1)}, \\
g_{i}^{(l)} & =\left(\sigma^{z}\right)_{i}^{(l)} .
\end{aligned}
$$

For an even number of legs we can also impose periodic boundary conditions on the rungs giving rise to a tube model as done in [5]. The rung interactions and magnetic field terms above commute with the Hamiltonians $(17,18)$ as a consequence of the fact that they can be expressed in terms of the symmetry algebra elements (đ).

It is important to mention that the Bethe Ansatz solutions of the models discussed above are all known. Using the Temperley-Lieb equivalence, the spectra of the above Temperley-Lieb models (in the absence of rung interactions and field terms) coincide with that of the $X X Z$ chain with the choice

$$
\Delta=-2^{(n-1)},
$$

where $\Delta$ is the $X X Z$ anisotropy. In this limit the Temperley-Lieb ladder models are thus massive for all $n$. For the Birman-Wenzl-Murakami class the solutions were originally obtained by Reshetikhin using the analytic Bethe Ansatz approach [13. More recently, these results have been rederived in an algebraic fashion by Martins and Ramos [14]. For the simplest case, that of the two-leg model corresponding to $s p(4)$ symmetry, the energy levels of the Hamiltonian are given by

$$
E=L+\sum_{i=1}^{M_{1}} \frac{1}{u_{i}^{2}-1 / 4}+J\left(M_{1}-L\right)+B\left(M_{1}-2 M_{2}\right)
$$

where the parameters $u_{i}$ are solutions of the Bethe Ansatz equations

$$
\begin{aligned}
-\left(\frac{u_{i}+1 / 2}{u_{i}-1 / 2}\right)^{N} & =\prod_{j=1}^{M_{1}} \frac{u_{i}-u_{j}+1}{u_{i}-u_{j}-1} \cdot \prod_{k=1}^{M_{2}} \frac{u_{i}-v_{k}-1}{u_{i}-v_{k}+1}, \quad j=1,2, \ldots, M_{1} \\
-1 & =\prod_{j=1}^{M_{1}} \frac{u_{j}-v_{i}-1}{u_{j}-v_{i}+1} \prod_{k=1}^{M_{2}} \frac{v_{k}-v_{i}+2}{v_{k}-v_{i}-2}, \quad i=1,2, \ldots, M_{2} .
\end{aligned}
$$

Above the parameters $M_{1}, M_{2}$ are restricted to the intervals

$$
\begin{aligned}
& 0 \leq M_{1} \leq 2 L, \\
& 0 \leq M_{2} \leq \min \left(M_{1}, L\right) .
\end{aligned}
$$

The expression for the energy (22) was derived using the the following rung basis states, in terms of which the rung interactions and field terms are diagonal,

$$
\begin{aligned}
|0\rangle & =\frac{1}{\sqrt{2}}(|\uparrow \downarrow\rangle-|\downarrow \uparrow\rangle), \\
|+\rangle & =|\uparrow \uparrow\rangle, \\
|-\rangle & =|\downarrow \downarrow\rangle, \\
| \pm\rangle & =\frac{1}{\sqrt{2}}(|\uparrow \downarrow\rangle+|\downarrow \uparrow\rangle) .
\end{aligned}
$$


These states may be interpreted as an empty site, occupation by a + or a - particle and a bound state, respectively. An advantage of this identifcation is that we may now write

$$
\begin{aligned}
M_{1} & =\sum_{i=1}^{L} n_{i}^{+}+n_{i}^{-}, \\
M_{2} & =\sum_{i=1}^{L} n_{i}^{-} .
\end{aligned}
$$

Note that the transformation $|0\rangle \leftrightarrow| \pm\rangle,|+\rangle \leftrightarrow|-\rangle, B \rightarrow-B, J \rightarrow-J$, is a symmetry of the system. Furthermore, the transformation $|0\rangle \leftrightarrow|-\rangle,|+\rangle \leftrightarrow| \pm\rangle, B \leftrightarrow J$ also leaves the model invariant.

It remains to investigate the thermodynamic properties of the ladder models. Without a magnetic field, it is easy to see that if $J$ is large and positive, $M_{1}=0$ for the ground state. In this case the system is completely empty and any excitation is massive. Since the model is critical at $J=0$, there is a phase transition at some critical value $J_{\mathrm{c}}$, similar to that in the $s u(4)$ case [4]. By a similar analysis it follows that for large but negative $J$ the system is massive again, but now completely filled. At negative $J$ there therefore exists another critical point, again similar to that of [4. In principle one should be able to calculate the location of these phase transitions exactly. However, little is known about the Bethe Ansatz solutions for the quantum spin chains associated with the orthogonal and symplectic Lie algebras.

\section{Acknowledgements}

This work is supported by the Australian Research Council. 


\section{References}

[1] E. Dagotto and T.M. Rice, Science 271 (1996) 618.

[2] H. Frahm and C. Rödenbeck, Europhys. Lett. 33 (1996) 47.

[3] H. Frahm and C. Rödenbeck, J. Phys. A: Math. Gen. 30 (1997) 4467.

[4] Y. Wang, Phys. Rev. B 60 (1999) 9236.

[5] M.T. Batchelor and M. Maslen, J. Phys. A: Math. Gen. 32 (1999) L377.

[6] S. Albeverio, S.-M. Fei and Y. Wang, Europhys. Lett. 47 (1999) 364.

[7] M.T. Batchelor and M. Maslen, Ground state energy and mass gap of a generalised quantum spin ladder, cond-mat/9907480.

[8] H. Fan, The Fermion ladder models: Extensions of the Hubbard model with $\eta$-pairing, cond-mat/9908028.

[9] H. Frahm and A. Kundu, Phase diagram of an exactly solvable $t-J$ ladder model, cond-mat/9910104.

[10] M.J. Martins and P.B. Ramos, J. Phys. A: Math. Gen. 27 (1994) L703.

[11] J. Birman and H. Wenzl, Trans. Am. Math. Soc. 313 (1989) 249; J. Murakami, Osaka J. Math. 241 (1987) 745.

[12] Y. Cheng, M.L. Ge and K. Xue, Commun. Math. Phys. 136 (1991) 195.

[13] N. Reshetikhin, Lett. Math. Phys. 14 (1987) 235.

[14] M.J. Martins and P.B. Ramos, Nucl. Phys. B500 (1997) 579. 